\title{
Numerical Analysis of Hub Effect on Hydrodynamic Performance of Propellers with Inclusion of PBCF to Equalize the Induced Velocity
}

\author{
Hassan Ghassemi, Assoc. Prof. \\ Amirkabir University of Technology \\ Amin Mardan, Ph. D. \\ Malekashtar University of Technology, Shahinshar-Isfahan, Iran. \\ Abdollah Ardeshir, Ph. D. \\ Amirkabir University of Technology, Tehran, Iran
}

\begin{abstract}
In this article the boundary element method (BEM) is applied to analyze the propeller hub as a non-lifting body and the blades in its vicinity as lifting bodies. In solver, the geometrical modeling of hub, blades are PBCF (Propeller Boss Cap Fin) constructed by quadrilateral elements. The velocity potential is determined on each element by discretized boundary integral equation. Iterative procedure is used to consider the adjacent body effect. In each step the body was independently analyzed with the influence of near body considered in inflow velocity. The induced velocity of propeller was calculated with and without PBCF in downstream. PBCF, an energy-saving device, reduces and uniforms the induced velocity of propeller in downstream. Numerical results of propeller hydrodynamic characteristics including hub effect, induced velocities, $P B C F$ influence are presented.
\end{abstract}

Keywords: propeller hub; induced velocities; hub vortex; PBCF

\section{INTRODUCTION}

The propeller is located at the stern of a ship where the inflow current is non-uniform due to boundary layer and wake formation. The propeller produces axial thrust from the engine power transmitted to it through a shaft. One of the augmentation tools for increasing thrust is the use of PBCF at the end of the hub, which causes to eliminate the hub vortex. The harmful effect of the hub vortex is twofold, firstly, it reduces the efficiency of the propeller and secondly, the rudder is exposed to corrosion because it is located downstream of the propeller where cavitation takes place.

Studies show that the performance of the propeller will degrade around the end portion of the hub. The vortices formed on the propeller's hub reduce the efficiency. The strength of the vortices depends on the axial load distribution on the propeller, and the geometry of the hub. The fins on the hub reduce cavitation of the hub and as a result the hydro-acoustic noise is reduced. In addition, the fins increase the efficiency of the propeller, especially in the case of controllable pitch propellers [1].

The effect of hub on hydrodynamic performance of the propeller is significant. There is some practical research on the hub size of the USS Barbey. In 1978, Wind [2] proposed a criterion for minimum hub diameter and suggested that the hub diameter of this ship should be increased from 1320 $\mathrm{mm}$ to $1500 \mathrm{~mm}$. He anticipated that the efficiency decreases proportionally to the decrease in surface area of the propeller's disc. The larger hub has greater hydrodynamic effect on blades and has to be considered in design process. A comparison between the efficiency of these two design situations gives important results.

Hess [3] employed source and dipole distribution for modeling blades and source distribution for modeling hub. In this study the lift force is displaced from intersection of the hub and blade to axis of rotation. This means zero angle of circulation in hub. In other words an extra strip from hub surface to hub center transfers a dipole with constant strength equal to the strength of the first strip on the blade. The hub vortex is formed along the axis of rotation and the vortex power is equal to sum of the power of all dipoles transferred from all blades. In 1991, considering the noise due to loading in stable conditions, Glegg showed that the hub effect on radiated noise of propeller in downstream is substantial [4]. In a research performed in 1992 on controllable pitch propellers it was shown that in addition to the pressure distribution in sections near the blade root the hub also affects the performance of the propeller in open-water condition [5]. According to the results obtained by Liu in 1995, in ordinary hubs of small diameter, which are mainly used in constant pitch propellers and surface ships, the effect of hub on hydrodynamic forces of the propeller such as thrust and torque, is not remarkable [6].

In 2009, Cai et al. [7] applied source and dipole singularities to analyze the propellers having nozzle including the effect of 
the hub. An iterative procedure was used to consider the effect of hub and blades on each other. In each step, hub and blades were independently analyzed with the influence of adjacent body considered in inflow velocity.

Now, the energy-saving propeller boss cap fins (PBCF) have been ordered for 2000 vessels worldwide. The PBCF is an energy-saving device attached to the propellers of vessel. It breaks up the hub vortex generated behind the rotating propeller (Fig. 1). Research and development studies on the PBCF started in 1986, and sales began the following year. Since then an increasing number of ship owners, mainly in Japan, began to

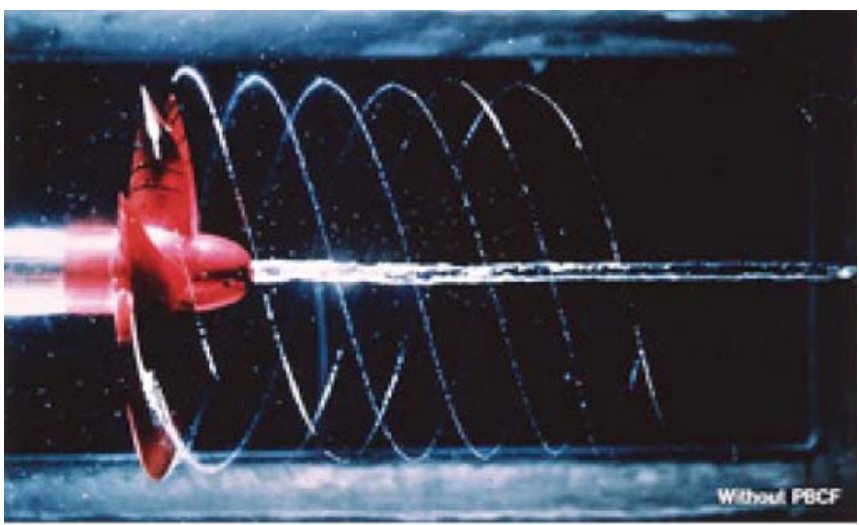

Before installation of PBCF

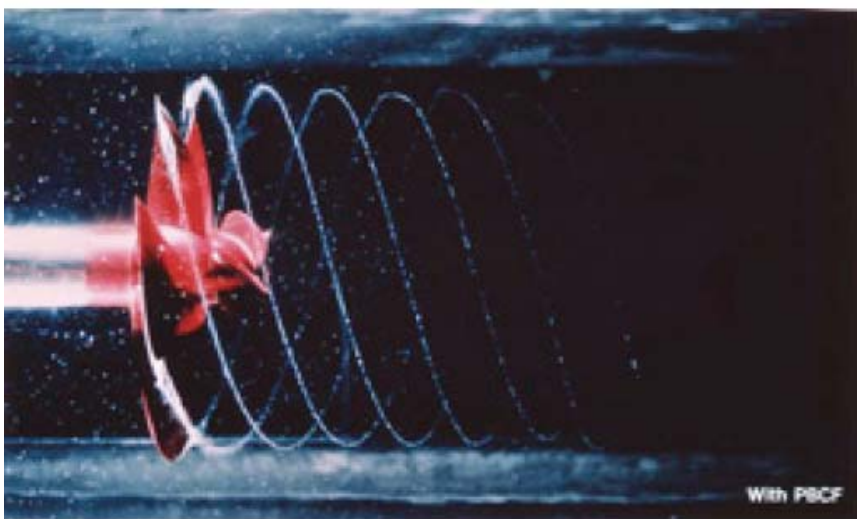

After installation of $\mathrm{PBCF}$

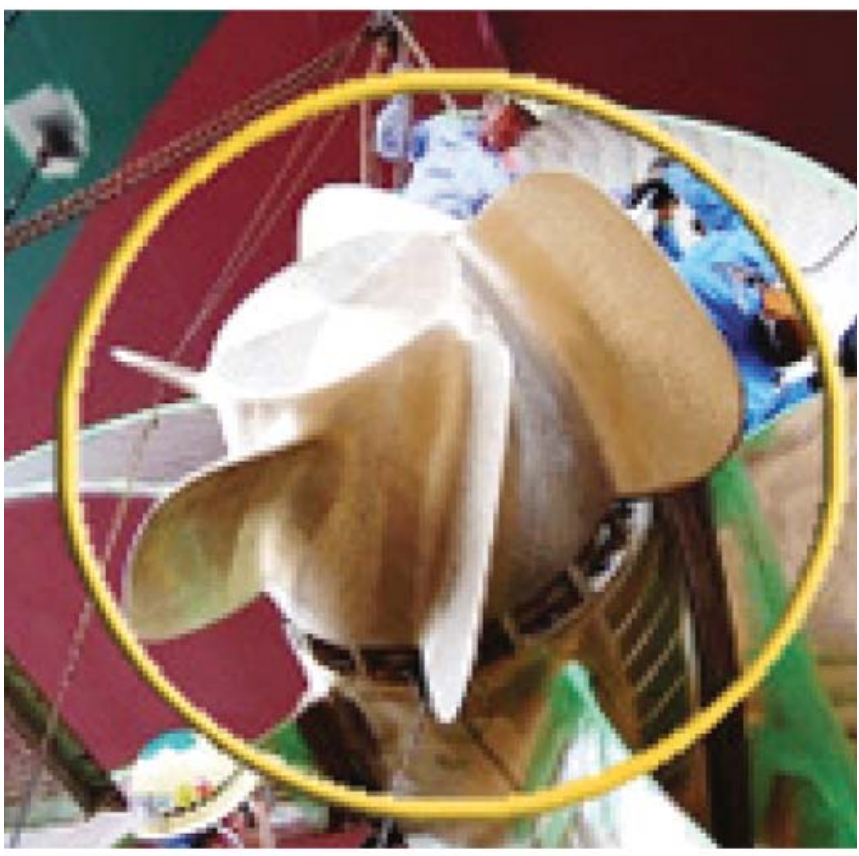

PBCF

Fig. 1. Hub vortex disappears after the installation of PBCF [8] adopt the system. In 1988 Ouchi first introduced it with the aim of increasing the efficiency and reducing energy loss due to hub vortex formation. Such vortex contains vapour bubbles that when burst, cause noise, corrosion and vibration in the system. By using fins, the vortices flowing on the hub are weakened and therefore the kinetic energy of rotational current is retrieved [9]. Ouchi et al. [10-12], during 1989 and 1992, extended and performed both numerical and experimental research on PBCF. They concluded that PBCF has significant effect on the energy saving. It not only causes to avoid the hub vortex but also increases the efficiency and reduce fuel consumption. Junglewitz reported that the use of PBCF results in hub vortex reduction and in consequence 2 to $5 \%$ increase in the efficiency [13]. Hsin et al. [14] presented a design procedure for a PBCF with the use of computational fluid dynamics (CFD).

The SPD (Ship Propeller Design) software code has been prepared by Ghassemi (the first author) and employed to the various propulsors such as propeller-rudder system (PRS) [15], high-skewed propeller [16], contra-rotating propeller [17] and surface piercing propeller [18], underwater vehicle under surface and submerged conditions [19]. In this code BEM is employed to perform the hydrodynamic analysis of marine propellers of all types. In June 2011 at the Second International Symposium on Marine Propulsors in Hamburg, Germany, the UK BMT Defence Services presented a paper reporting on 'a before and after' speed test on an Aframax ship operated by a major firm. This showed energy saving of about 4\% [20].

In this article, the presence of the fins in induced downstream effects of the propeller is analyzed. The performance of the fins at the end of the hub is evaluated by changing two parameters: the angle of installation of the fin on the end of the hub and the phase angle between propeller and the fin. In addition, the effect of hub diameter and its conical angle which are common parameters in hydrodynamic design of hull and propeller, is studied. The following sections are organized as follows: modeling the PBCF is explained in Sec. 2, governing equations are described in Sec. 3, numerical results and discussions are presented in Sec. 4. Finally, the conclusions are given in Sec. 5.

\section{MODELING THE PBCF}

In geometrical design of PBCF the following points have to be noted [11]:

- The number of fins should be equal to the number of blades of the propeller.

The phase difference between the cross-section of the blade root and the fins varies from -20 to 30 degrees.

The diameter of the fins should not exceed $33 \%$ of the propeller's diameter.

The leading edge of the fins is located between the roots of two adjacent blades.

The first point is because each fin has to reduce the wake of one blade. The inflow velocity, rotational speed (rpm) of the propeller and the angle of attack of blade cross section determines the second point. In formulating Point 3 , it is noted that increasing the surface area of the fins produces torque and reduces the efficiency of the propeller, therefore a limitation on diameter is imposed. Total impact of the wake due to the fin surface is considered in Point 4. Including all these parameters into design procedure is rather complicated. In this research a technique was attempted to construct a model of PBCF with the use of the above-mentioned points. Fig. 2 shows a model of PBCF with trailing vortex wake. The total number of the elements is the summation of elements on blades, hub and 
fins. The total numbers of elements are equal to 8500 , i.e. 2400 located on blades, 2400 on fins and 3500 on the hub. The main parameters of the propeller and the fin are listed in Tabs. 1 and 2.

Tab. 1. Main dimensions of the propeller

\begin{tabular}{|c|c|}
\hline Parameter & Value \\
\hline Number of blades & 4 \\
\hline Diameter [m] & 1.0 \\
\hline Pitch ratio (at 0.7R) & 1.084 \\
\hline Expanded Area Ratio (EAR) & 0.5 \\
\hline Skew [deg.] & 10 \\
\hline Rake [deg.] & 6 \\
\hline Direction of rotation & Clockwise \\
\hline Propeller type & MAU \\
\hline
\end{tabular}

Tab. 2. Main parameters of the $P B C F$

\begin{tabular}{|c|c|}
\hline Parameter & Value \\
\hline Number of fins & 4 \\
\hline Diameter [m] & 0.33 \\
\hline Angle of installation & variable \\
\hline Chord of fin & variable \\
\hline Direction of rotation & clockwise \\
\hline
\end{tabular}

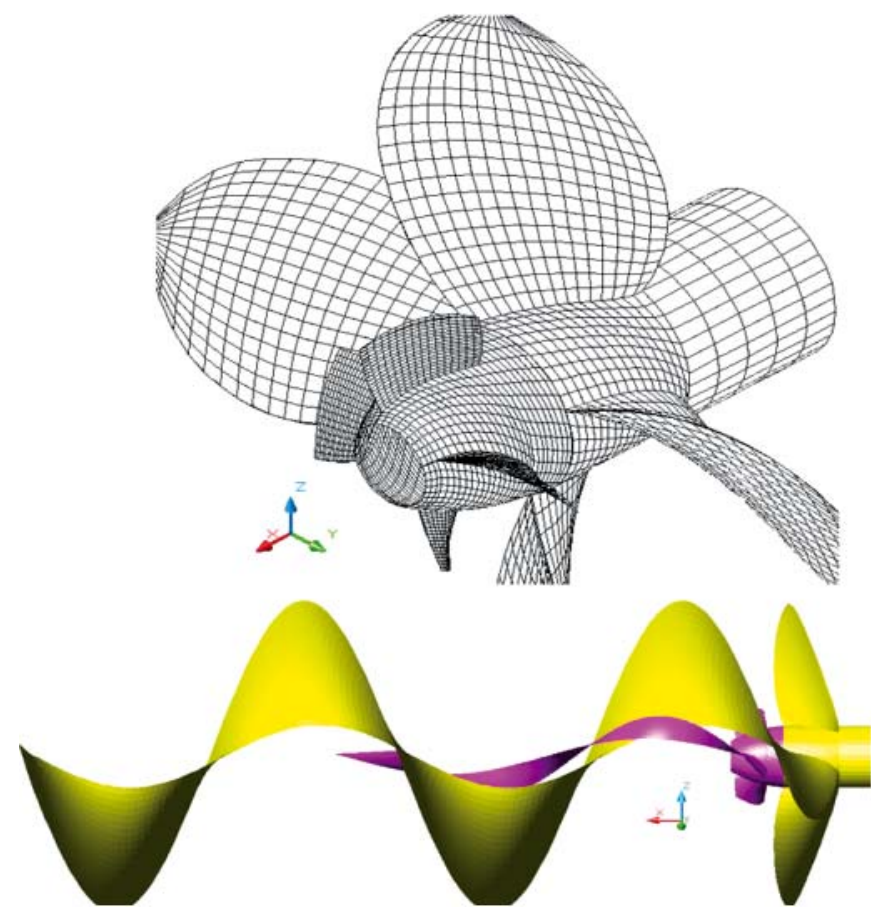

Fig. 2. The PBCF model along with trailing vortex wake

\section{GOVERNING EQUATIONS}

As assumed, the flow is considered inviscid, incompressible and irrotational around the body. The assumptions lead to a boundary value problem for the velocity potential with the Laplace equation satisfied in the fluid domain.

$$
\nabla^{2} \Phi(\mathrm{x}, \mathrm{y}, \mathrm{z})=0
$$

The potential equation is an elliptical equation and for its solution, we need to define $\Phi$ on the boundary or its derivative $\partial \phi / \partial \mathrm{n}$ in the perpendicular direction to the boundary. On the surface of the body, velocity component perpendicular to the element boundary should be zero. Therefore, the Neumann boundary condition called kinematic boundary condition could be used. This states that the total velocity normal to surface is zero.

$$
\begin{gathered}
\Phi=\emptyset+\emptyset_{\text {in }} \\
\Rightarrow \frac{\partial \Phi}{\partial n}=0 \Longrightarrow \frac{\partial \emptyset}{\partial n}+\frac{\partial \emptyset_{\text {in }}}{\partial n}=0 \\
\Rightarrow \frac{\partial \emptyset}{\partial n}=-\emptyset_{\text {in }}=-V_{0} \cdot \vec{n}
\end{gathered}
$$

where:

$\vec{n} \quad-\quad$ the normal vector of the surface.

The thickness of the wake surface is assumed zero. The normal velocity difference and the transverse pressure difference is zero, although the potential difference could be present.

$$
\begin{gathered}
(\Delta p)_{\text {on } S_{w}}=p^{+}-p^{-}=0 \\
\left(\Delta \frac{\partial \phi}{\partial n}\right)_{o n S_{w}}=\left(\frac{\partial \phi}{\partial n}\right)^{+}-\left(\frac{\partial \phi}{\partial n}\right)^{-}=0
\end{gathered}
$$

For the lifting bodies in steady state, the potential difference in transverse direction to the wake surface, is equal to the circulation around the body and is constant along the streamline on the wake surface.

$$
(\Delta \phi) \operatorname{onS}_{\mathrm{w}}=\phi^{+}-\phi^{-}
$$

To uniquely determine the circulation on the trailing edge of the blade, the Kutta condition is used. Generally, this condition states that the flow velocity remains constant at the trailing edge of the blade.

$$
|\nabla \phi|_{\text {T.E. }}<\infty
$$

On the external control surface, in the case that this surface is located at infinite distance from the body, the disturbance velocity created by the body should vanish.

$$
\nabla \phi \rightarrow 0\left\{\mathrm{~S}_{\infty} \rightarrow \infty\right\}
$$

\section{THE BOUNDARY ELEMENT METHOD}

The boundary value problems of velocity potential outside the body surface could be converted to an integral equation:

$$
\begin{gathered}
2 \pi \phi(p)=\iint_{S_{B}}\left[\phi(q) \frac{\partial}{\partial n_{q}} \frac{1}{R(p ; q)}-\frac{\partial \phi(q)}{\partial n_{q}} \frac{1}{R(p ; q)}\right] d S+ \\
\quad+\iint_{S_{W}} \Delta \phi(q) \frac{\partial}{\partial n_{q}} \frac{1}{R(p ; q)} d S
\end{gathered}
$$

Equation (7) is the second- type Fredholm integral equation for the variable $\phi$, since $\partial \phi / \partial \mathrm{n}$ is known on the surface of the body. The potential difference in transverse direction through the wake surface is equal to the potential difference of the top and bottom surfaces of the blade at the trailing edge. Discretizing the equation (7) results in a linear system of equations with the variable $\phi$. The surface velocity and, as a result, the pressure on the surface of the body could be obtained by numerical differentiation of the calculated potential distribution. $R(p ; q)$ 
is the distance of the point $\mathrm{p}(\mathrm{x}, \mathrm{y}, \mathrm{z})$ from the point $\mathrm{q}(\xi, \eta, \zeta)$. It is expressed as follows.

$$
\begin{gathered}
R=|Q-P|= \\
=\sqrt{(\xi-x)^{2}+(\eta-y)^{2}+(\zeta-z)^{2}}
\end{gathered}
$$

The induced velocities in a field point $\mathrm{p}$ outside the body surface, is obtained by the gradient of the velocity potential $\phi$.

$$
\begin{gathered}
v(p)=\nabla_{p} \phi(p)= \\
=\frac{1}{4 \pi} \iint_{S_{B}}^{\cdot} \phi(q) \nabla_{p} \frac{\partial}{\partial n_{q}} \frac{1}{R(p, q)} d S+ \\
+\frac{1}{4 \pi} \iint_{S_{W}}^{\cdot} \Delta \phi(q) \nabla_{p} \frac{\partial}{\partial n_{q}} \frac{1}{R(p, q)} d S+ \\
-\frac{1}{4 \pi} \iint_{S_{B}}\left(V_{I} \cdot n_{q}\right) \nabla_{p} \frac{1}{R(p, q)} d S
\end{gathered}
$$

\section{CALCULATION OF THE HYDRODYNAMIC PERFORMANCE}

By using finite difference method, the potential derivatives in radial direction and along chord length could be determined and the velocity distribution on the propeller's surface could be computed. The criteria for evaluating the performance of a propeller are: thrust coefficient, torque coefficient and efficiency which are determined according to tangential velocity distribution on the surface.

$$
\begin{gathered}
C_{p}(p)=1-\frac{\left|V_{I}\right|^{2}}{\left|V_{T}\right|^{2}} \\
P\left(p_{i}\right)=\frac{1}{2} \rho\left[\left|V_{I}\right|^{2}-\left|V_{T}\right|^{2}\right] \\
T_{\text {Potential }}=K \sum_{i=1}^{N_{B}} P\left(p_{i}\right) n_{x i} \cdot \Delta S_{i} \\
Q_{\text {Potential }}=K \sum_{i=1}^{N_{B}} P\left(p_{i}\right)\left(n_{y i} \cdot z_{i}-n_{z i} \cdot y_{i}\right) \cdot \Delta S_{i}
\end{gathered}
$$

The element area, normal vector components $\left(\mathrm{n}_{\mathrm{x}}, \mathrm{n}_{\mathrm{y}}, \mathrm{n}_{\mathrm{z}}\right)$, coordinates of the center of the element $(\mathrm{x}, \mathrm{y}, \mathrm{z})$ and number of blades $(Z)$ are the geometrical parameters which influence the thrust and torque of the propeller in potential flow. For determining the viscosity effects, its drag coefficient is determined experimentally:

$$
\begin{gathered}
V_{j}=\sqrt{V_{A}^{2}+\left(2 \pi n r_{j}\right)^{2}}, \quad j=1,2,3, \ldots, m \\
R n_{j}=\frac{(\text { chord } \times V)_{j}}{v} \\
\text { Force }_{j}=0.5 \rho\left(C_{f} \times{\text { chord } \left.\times V^{2}\right)_{j}}^{m} T_{\text {viscous }}=K \sum_{i=1}^{m} \text { Force }_{j} \cdot \sin \beta_{j}\right. \\
Q_{\text {viscous }}=K \sum_{i=1}^{m} r_{j} \cdot \text { Force }_{j} \cdot \cos \beta_{j}
\end{gathered}
$$

Total thrust and torque are the sum of viscous component and potential components.

$$
\begin{gathered}
T=\left|T_{\text {Pot }}\right|+\left|T_{\text {Vis }}\right| \\
Q=\left|Q_{\text {Pot }}\right|-\left|Q_{\text {Vis }}\right|
\end{gathered}
$$

Finally, the hydrodynamic characteristics of the propeller can be defined as:

$$
\begin{gathered}
J=\frac{V_{A}}{n D}, \quad \eta=\frac{K_{T}}{K_{Q}} \frac{J}{2 \pi} \\
K_{T}=\frac{T}{\rho n^{2} D^{4}}, \quad K_{Q}=\frac{Q}{\rho n^{2} D^{5}}
\end{gathered}
$$

where:

$\rho-$ density of water,

$v$ - kinematic viscosity,

$\mathrm{n}$ - rotational speed of the propeller

D - diameter of the propeller.

\section{NUMERICAL RESULTS AND DISCUSSION}

\section{Effect of hub diameter}

In this section the effect of increasing hub diameter with respect to propeller diameter is investigated for constant - diameter and adjustable - diameter propellers. The relative percent change of the hydrodynamic coefficients with and without hub is obtained from the following equation:

$$
\% \text { Delta }=\frac{\text { Hydro Coeff } \cdot(\text { without Hub-with Hub })}{\text { Hydro Coeff } \cdot \text { without Hub }} \times 100
$$

Fig. 3 shows the relative percent change of the propeller coefficients versus hub ratio. In the first case, the diameter of the propeller is constant while the diameter of the hub is increasing. In the second case, the diameter of the hub and propeller has been increased with the same ratio. The results show that the hub effect will reach its minimum at a certain hub ratio. On the other hand, the percent change in hydrodynamic coefficients is equal in both cases. The reason that percent change remains constant in the two cases is that the expanded area ratio, the number of blades, pitch of the propeller and hub ratio have remained constant. Comparing the change in circulation distribution in radial direction due to change in hub ratios, it could be seen that in case of low hub ratio, the

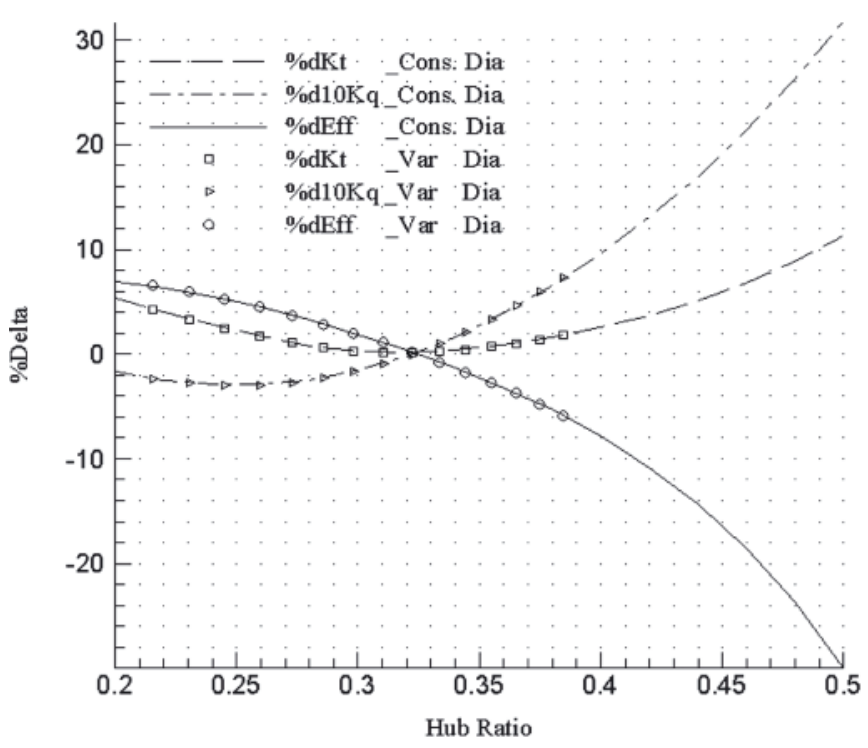

Fig. 3. Relative percent change of the propeller coefficients versus hub ratio 
effect of hub is limited to the radial cross-sections near to the root but with increasing hub ratio, the outer cross-sections also become affected.

Thrust and torque changes with different hub ratios in cases of constant and variable diameter are shown in Figs. 4 and 5,
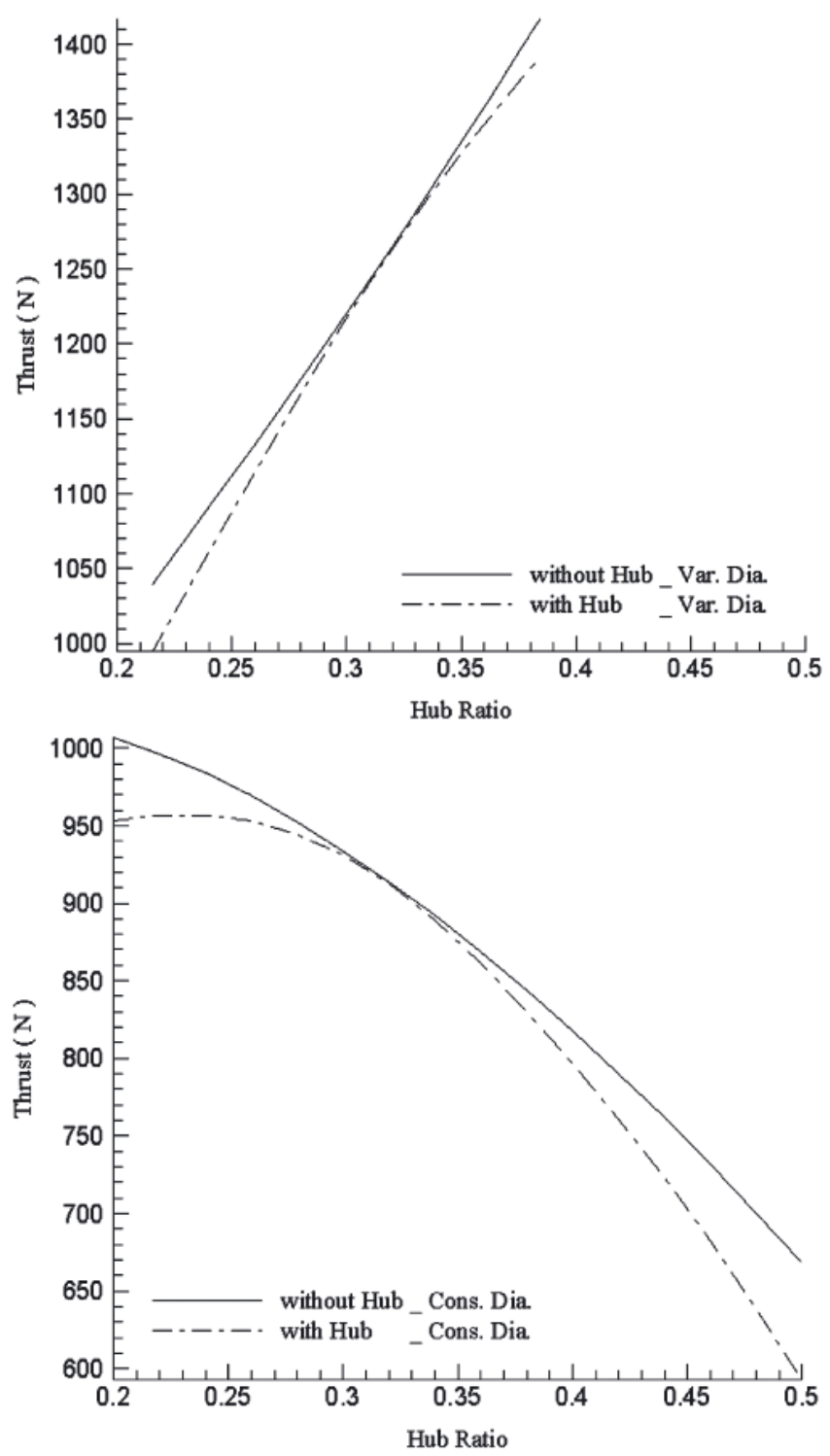

Fig. 4. Thrust change with different hub ratios for constant and variable diameter cases

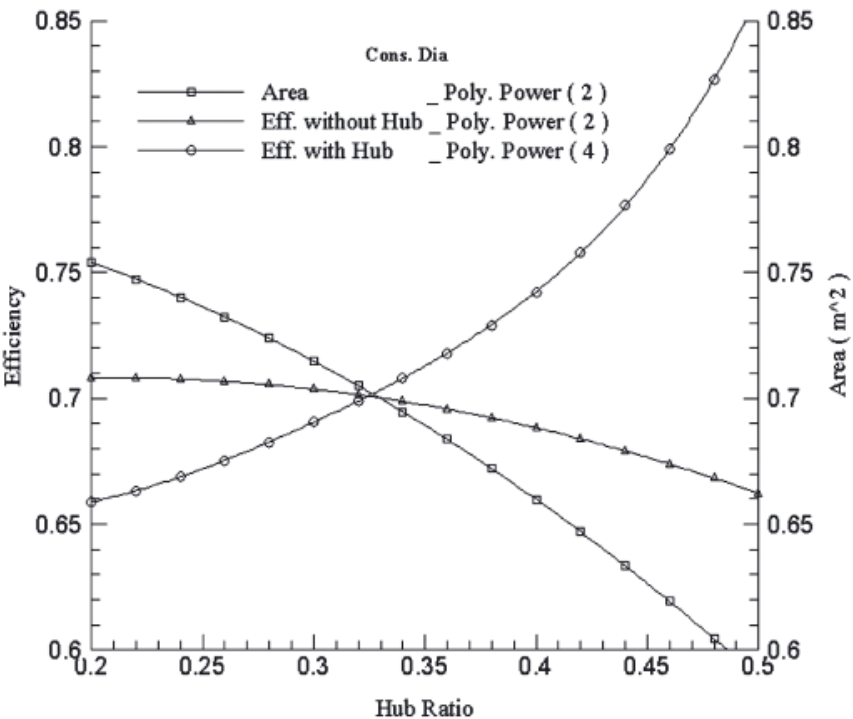

respectively. In the case of constant diameter, due to decrease in the space between the root and tip of the propeller, the thrust and torque decrease, but in the case of variable diameter, this space is increasing with a constant factor and it seems logical that the output curves of the propeller are ascending.
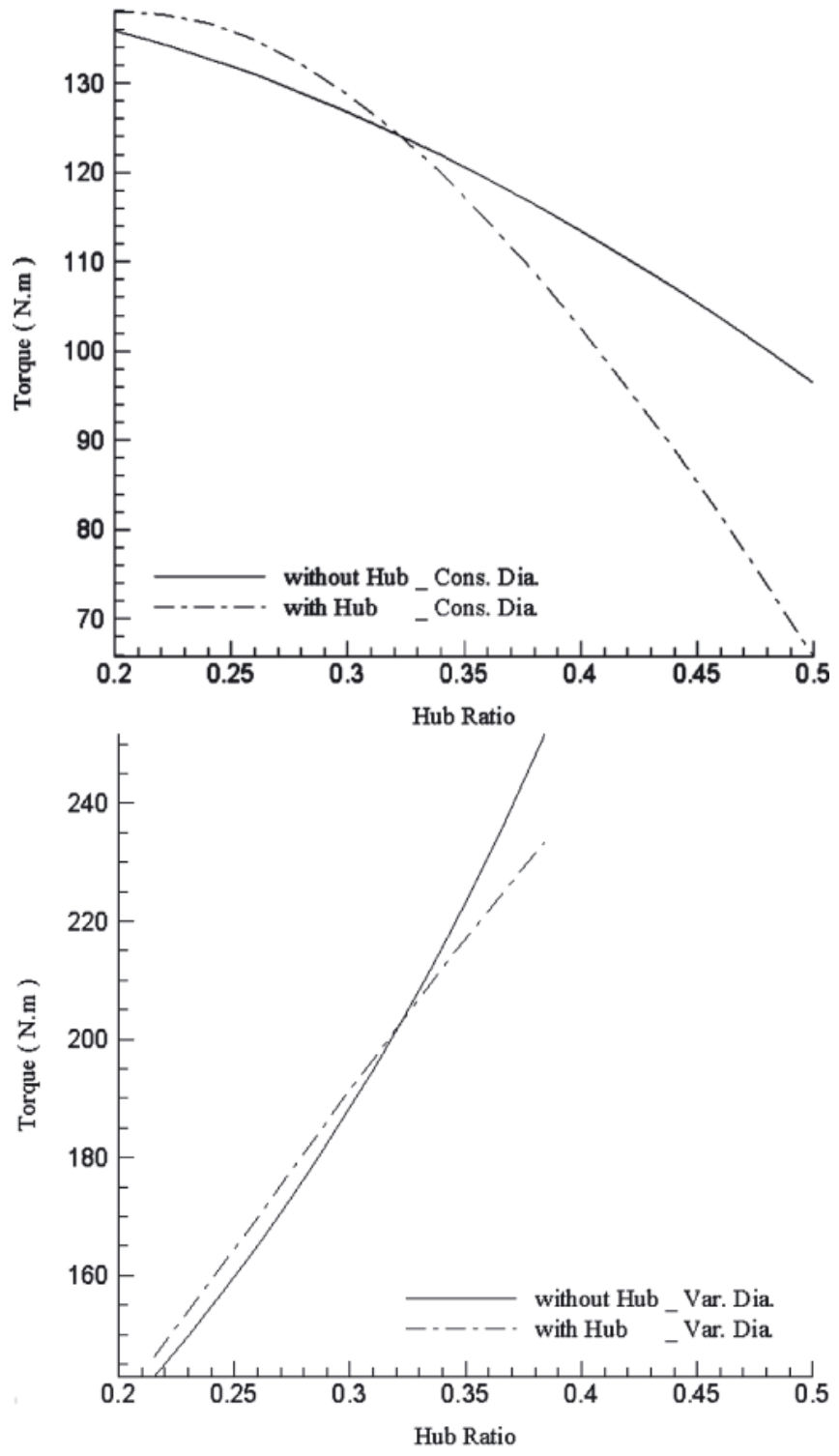

Fig. 5. Torque change with different hub ratios for constant and variable diameter cases

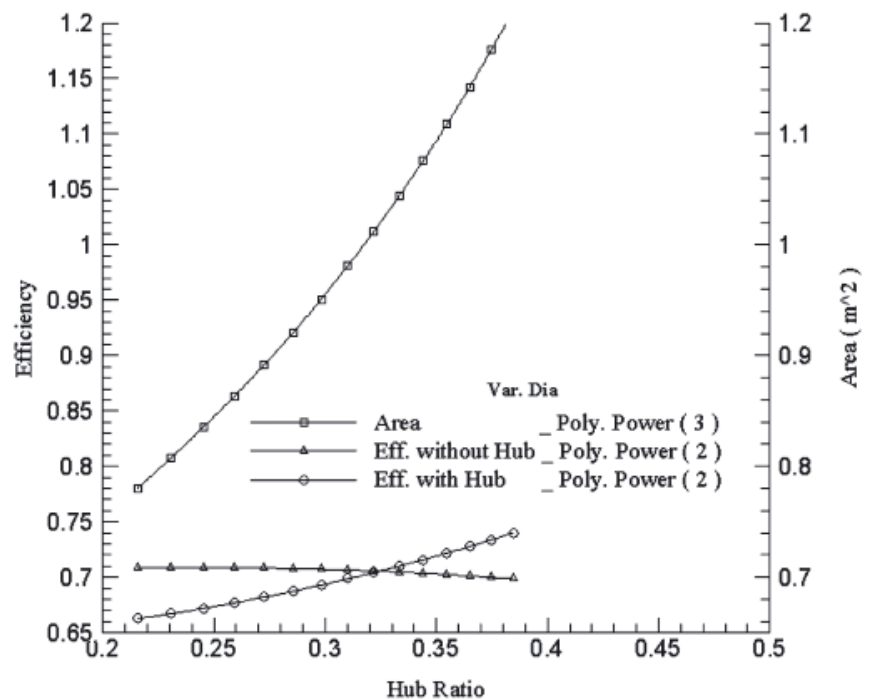

Fig. 6. The effect of hub on efficiency change for constant and variable diameter cases 
In the constant diameter case, the hub has significant effect on propeller's efficiency such that the order of the polynomial proportional to efficiency change has risen by two (Fig. 6). In the variable diameter case, although the hub has changed the performance of the propeller, the order of the polynomial has not changed.

\section{Effect of the conical angle of hub}

The conical angle may change from zero to 15 degrees at three different hub ratios. Fig. 7 shows the change in hydrodynamic coefficients versus conical angle of hub at two hub ratios (0.2 and 0.4). At both hub ratios, when the conic angle of the hub increases, the change in the hydrodynamic coefficients increases. In the case of 0.3 hub ratio, the thrust and torque coefficients increase more while the efficiency is changed slightly.

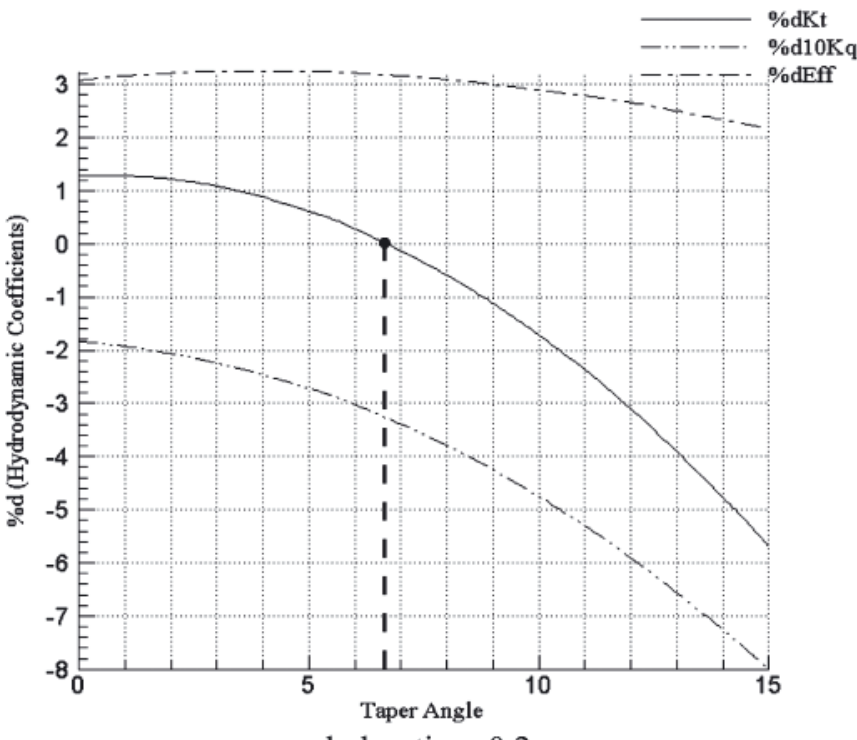

hub ratio $=0.2$

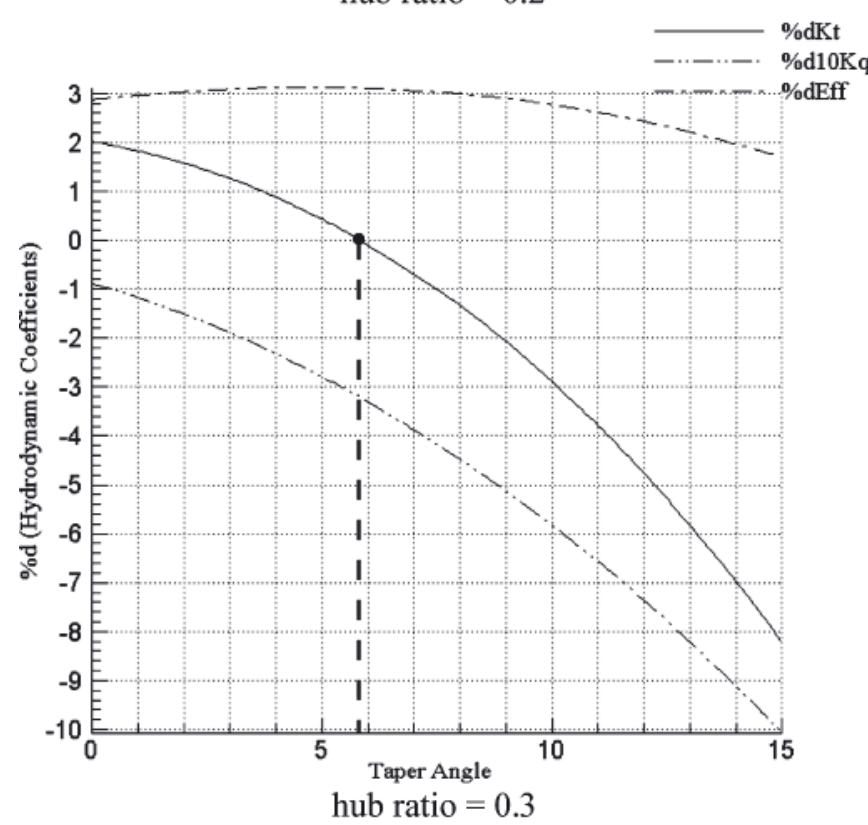

Fig. 7. Change in the performance coefficients along with increasing the hub angle

\section{Analyzing the performance of $\mathrm{PBCF}$}

In this section, besides analyzing the hydrodynamic effect of the fin, the change in its geometrical parameters is studied. Generally, the effect of the fins is to reduce the induced velocity which in turn reduces the hub vortex. Here, the effect of the fin at the end of the hub and the effect of its position and angle on the induced velocity is examined.

\section{A.Overall analysis of the $\mathrm{PBCF}$}

PBCFs are not considered thrust producing devices. Their purposes are to reduce and distribute hub vortex into bigger core at the downstream where it is spread out. Fig. 8 shows axial induced velocity contour at downstream position, $x / R_{\mathrm{PBCF}}$ $=0.5$, with and without fins. It may be seen that the fins caused significant reduction in maximum induced velocity and more uniform distribution of induced velocities.

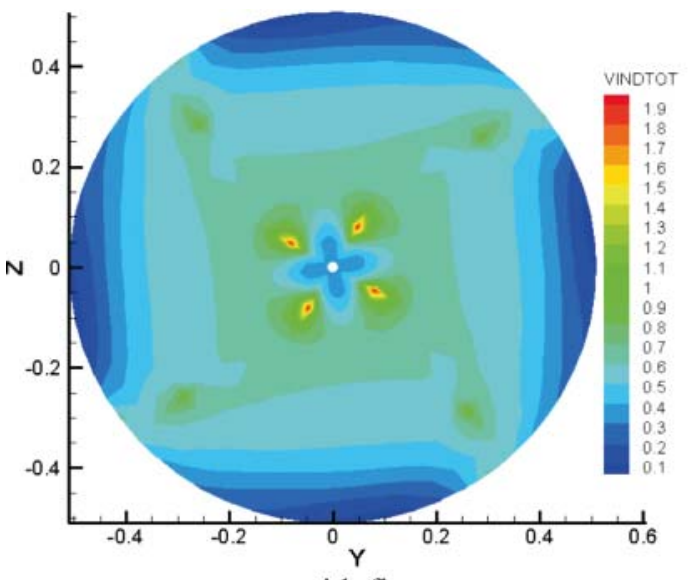

with fin

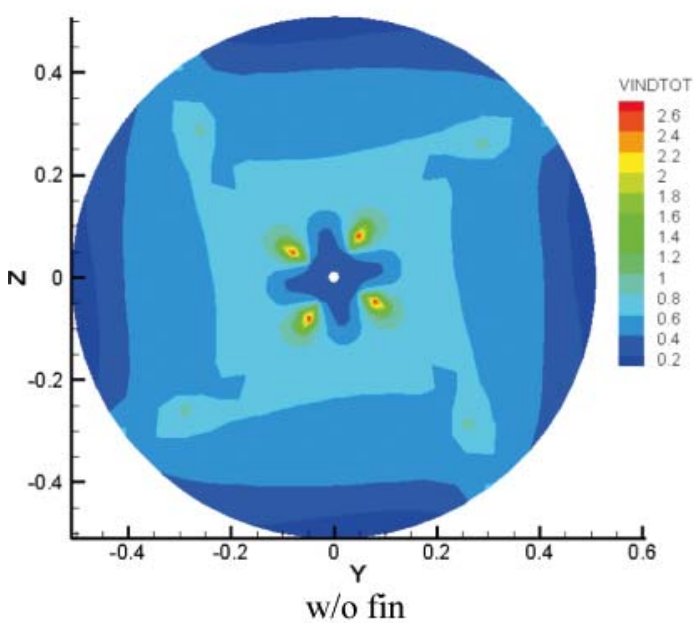

Fig. 8. Axial induced velocity contour at downstream position, $x / R_{P B C F}=0.5$

The change in induced effects of the propeller in axial direction is shown in Fig. 9. Without fins, the induced effects are conveyed downstream with a certain period. This periodic feature is related to the formation of flow near the end of the hub, which is proportional to propeller's rotational speed. Installing fins at the end of the hub minimizes these effects.

The most important effect of the fins is reduction of negative or backward components of the induced effects in axial direction. As the distance from the propeller increases the effects become reduced.

B. Effect of phase difference between the blade and fin

Fig. 10 shows the change in phase difference between the fin and propeller. The fin should be so placed that its leading edge lies between two blades, so that it has the best performance. This is analyzed by changing the phase difference between the fin and the blades. Fig. 11 shows the change in axial component of the induced velocities as the angular position of the fin is changing (phase difference of $\pi / 14$ and $6 \pi / 14$ ) at the axial 


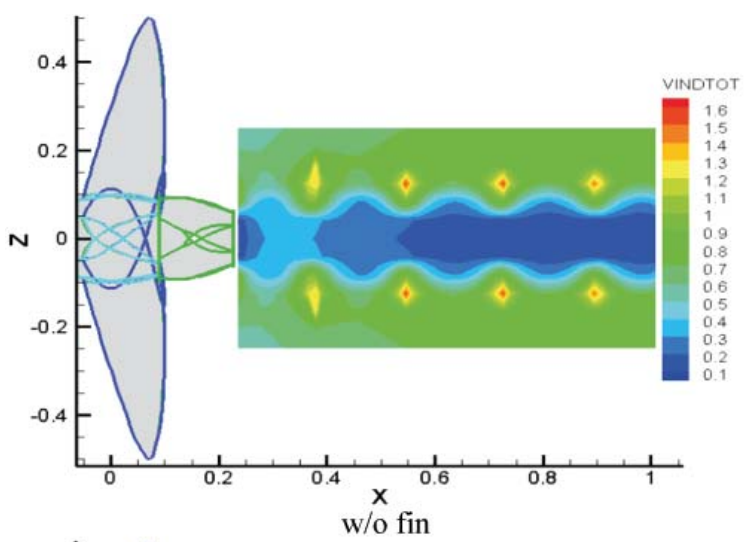

ratios of 1 and 2. Fig. 13 shows the axial induced velocity at the two pitch ratios. In these calculations, we were seeking the effect of the angle on the axial induced velocity. The greater the angle or pitch the greater the effect of induced velocity.
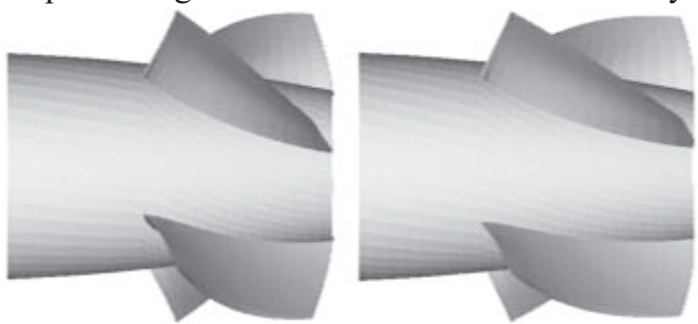

Fig. 12. The change in angle of installation of the fin
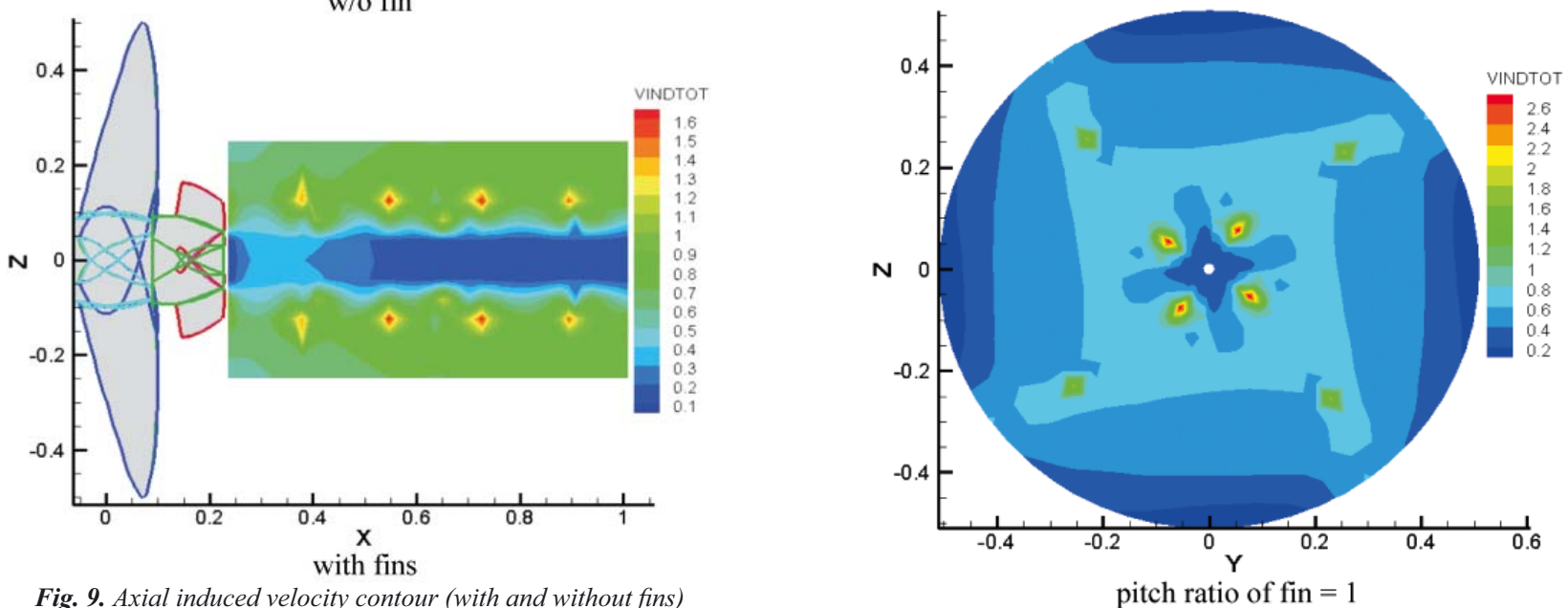

distance of $\mathrm{x} / \mathrm{R}_{\mathrm{PBCF}}=0.5$ from the end of the propeller. By changing the angular position of the fin, the negative component of the induced velocities has increased more.

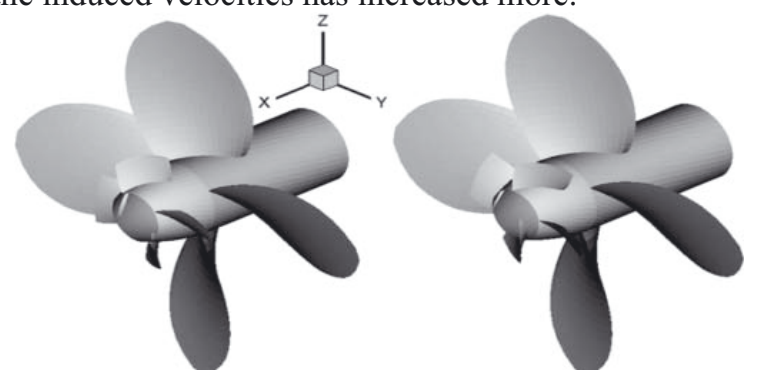

Fig. 10. The change in phase difference between the fin and propeller

\section{C.Effect of fin installation}

Angle of installation is one of the geometrical parameters of the fin, which is related to the pitch angle. The change in the angle of installation of the fin is plotted in Fig. 12 for two pitch

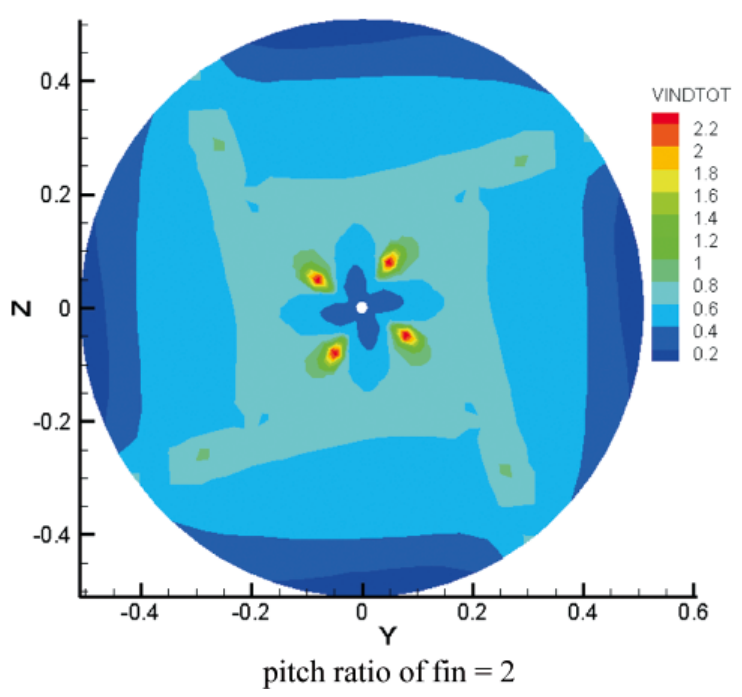

Fig. 13. Axial induced velocity of the propeller at two pitch ratios of fin
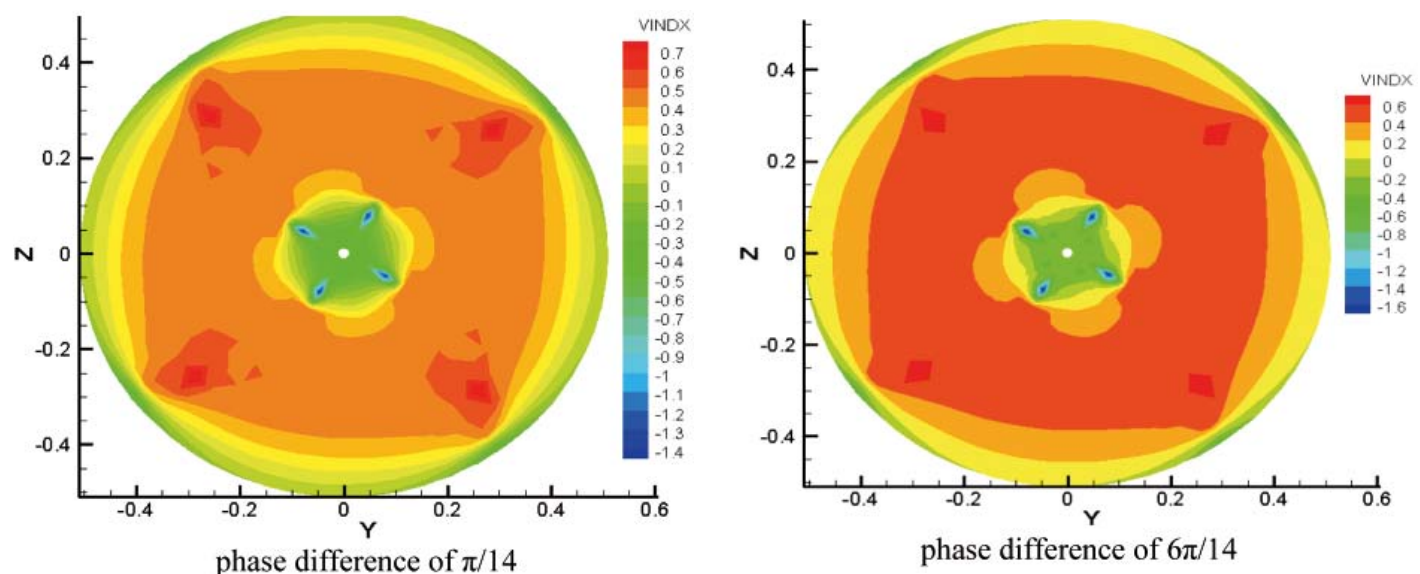

Fig. 11. Change of the axial component of the induced velocity at downstream position, $x / R_{P B C F}=0.5$ 


\section{CONCLUSIONS}

In this article, the effect of hub and PBCF on hydrodynamic performance has been studied.

From the numerical results the following conclusions can be drawn:

- By increasing the hub ratio, thrust will be reduced and torque increased,which causes the efficiency to decrease.

- In a propeller with constant diameter, increasing the hub ratio has a significant effect on its performance. This change in performance could be minimized if the diameter of the propeller is increased proportionally to the hub diameter.

- At each hub ratio, increasing the conical angle of the hub has no effect on efficiency of the propeller.

- Placing the fins at the boss causes the downstream induced effects of the propeller to decrease and makes downstream flow more uniform.

- The fins reduce the negative component of the induced effects of the propeller and have only a slight effect on the components in the direction of the flow.

- The angle of installation of the fin and its phase difference against the propeller are two important items to be considered in geometrical modeling of the fin, which, if chosen incorrectly, could significantly degrade its performance.

\section{NOMENCLATURE}

$\mathrm{C}$ - chord length at each radius

$\mathrm{C}_{\mathrm{f}}$ - friction coefficient

$\mathrm{C}_{\mathrm{p}}$ - pressure coefficient

D - propeller's diameter

J - Jaccobian transform

$\mathrm{J}$ - advance velocity ratio

$\mathrm{K}_{\mathrm{T}} \quad-$ thrust coefficient of the propeller

$\mathrm{K}_{\mathrm{Q}}$ - torque coefficient of the propeller

$\vec{n}$ - normal vector of the surface

$\mathrm{P} / \mathrm{D}$ - pitch-diameter ratio

Q - total torque of the propeller

$\mathrm{Q}_{\text {Pot }}$ - potential component of the propeller torque

$\mathrm{Q}_{\mathrm{Vis}}$ - viscous component of the propeller torque

$\mathrm{r}_{\mathrm{h}} \quad$ - hub radius

$\mathrm{R}_{\mathrm{n}} \quad$ - Reynolds number at radius $\mathrm{r}$

$\mathrm{S}_{\mathrm{B}}$ - blade surface

$\mathrm{S}_{\mathrm{w}}$ - trailing wake surface

$\mathrm{T} \quad$ - total propeller thrust

$\mathrm{T}_{\mathrm{Pot}}$ - potential component of the propeller thrust

$\mathrm{T}_{\mathrm{Vis}}$ - viscous component of the propeller thrust

$\mathrm{V}_{\mathrm{I}} \quad$ - inflow velocity to the propeller

$\vec{V}_{I}-$ total tangential velocity on propeller's surface

$\mathrm{Z}$ - number of blades

$\Delta \mathrm{S}_{\mathrm{i}}$ - surface area of each element

$\Phi \quad$ - total velocity potential

$\phi \quad-$ perturbation velocity potential

$\phi_{\text {in }} \quad-$ inflow velocity potential

$\eta \quad$ - propeller efficiency

\section{BIBLIOGRAPHY}

1. Wang, H.: Hub effects in propeller design and analysis. $\mathrm{PhD}$ Dissertation, Ocean Engineering Department, MIT, 1985.

2. Wind, J.: Hub size selection criteria for controllable pitch propellers as a means to ensure system integrity. Naval Engineers Journal, No. 90(4), 1978, pp.49-61

3. Hess, J.L.: Calculation of potential flow about arbitrary threedimensional lifting bodies. McDonnell Douglas Rep. MDC J5679-01, 1972.

4. Glegg. S.A.: Effect of center body scattering on propeller noise. AIAA, vol. 29, April 1991, p. 572-576
5. Yang, C.,Wang G., Masahiro T.: A method of improving the precision of the prediction of the performance characteristics of a CPP the effect of boss being taken into consideration. Shipbuilding of China, No. 116, 1992

6. Liu, Y-H, Ikehata, M.: Investigation into hub effect of marine propeller by surface vortex lattice method. Journal of Society of Naval Arch. Japan, Vol. 177, 1995, pp. 59-66

7. Cai, H. P., Su Y. M., Li X., Shen H.L.: Using the surface panel method to predict the steady performance of ducted propellers. J. Marine. Sci. Appl., 2009.

8. Http://www.mol.co.jp/pr-e/2011/e-pr_1145.html

9. Ouchi, K.B., Kono Y., Shiotsu T., Koizuka H.: PBCF (Propeller Boss Cap Fins). J Soc. Nav. Arch. Japan (SNAJ),Vol 163, 1988.

10.Ouchi, K., Tamashima, M.: Research and development of PBCF (propeller boss cap fin), new and practical device to enhance propeller efficiency. $4^{\text {th }}$ international Symposium on Practical Design of Ships and Mobile Units (PRADS), Bulgaria,1989

11.Ouchi, K., Tamashima M., Arai K.: Reduction of Propeller cavitation Noise by PBCF (Propeller Boss Cap Fins). Kansai Soc. Naval Arch. Japan (KSNAJ), Vol. 216, 1991.

12.Ouchi, K., Tamashima M.: A study on correlation between propeller pitch distribution and improvement of propeller efficiency by PBCF. Kansai Soc Naval Arch Japan (KSNAJ), Vol. 217, 1992

13.Junglewitz, A.: Der Nabene influße im Schrauben propeller. PhD Thesis, Univ. of Rostock., 1996.

14.Hsin, C.Y., Lin, B.H. and Lin, C.C.: The optimum design of a propeller energy-saving device by computational fluid dynamics. The Proceeding of the Fifth Int. Conf. of Computational Fluid Dynamics (ICCFD), Korea, 2008, pp.655660

15.Ghassemi, H., Ghadimi, P.: Computational hydrodynamic analysis of the propeller-rudder and the AZIPOD systems. Ocean Engineering, Vol. 34, 2007, pp.117-130

16. Ghassemi, H.: The effect of wake flow and skew angle on the ship propeller performance. Scientia Iranica, No. 2,Vol. 16, 2009, pp.149-158

17.Ghassemi, H.: Hydrodynamic performance of coaxial contrarotating propeller (CCRP) for large ships. Polish Maritime Research, No. 59,Vol. 16, 2008, pp.22-28

18. Ghassemi, H.: Hydrodynamic characteristics of the surfacepiercing propellers for the planing craft. J. Marine Science \& Application (JMSA), Vol. 7, 2008, pp.147-156

19.Ghassemi, H., Ghadimi, P.: Hydrodynamic efficiency improvement of the high skew propeller for the underwater vehicle under surface and submerged conditions. J. Ocean Univ. China (JOUC), Vol. 10, 2011, pp.314-324

20.Richard, H. H., Dinham-Peren, T., Nojiri, T.: Model and full scale evaluation of a 'Propeller Boss Cap Fins'device fitted to an Aframax tanker. Second International Symposium on Marine Propulsors, SMP'11, Hamburg, Germany, June 2011.

\section{CONTACT WITH THE AUTHORS}

Hassan Ghassemi, Assoc. Prof.

Faculty of Marine Technology

Amirkabir University of Technology

Hafez Ave.,

Tehran, IRAN

e-mail: gasemi@aut.ac.ir

Amin Mardan, Ph. D.

Faculty of Marine Engineering,

Malekashtar University of Technology, Shahinshar-Isfahan, IRAN

Abdollah Ardeshir, Ph. D.

Faculty of Civil \& Environmental Engineering, Amirkabir University of Technology,

Hafez Ave.,

Tehran, IRAN 